\title{
ON THE RANK OF A SPACE
}

\author{
BY \\ CHRISTOPHER ALLDAY
}

\begin{abstract}
The rank of a space is defined as the dimension of the highest dimensional torus which can act almost-freely on the space. (By an almost-free action is meant one for which all the isotropy subgroups are finite.) This definition is shown to extend the classical definition of the rank of a Lie group. A conjecture giving an upper bound for the rank of a space in terms of its rational homotopy is investigated.
\end{abstract}

1. Introduction. Various attempts have been made to define the rank of a space. The motive for these definitions has been a desire to extend the classical definition of the rank of a compact Lie group. The latter is the dimension of a maximal torus in the group, and it finds algebraic expression in the following results of Hopf and Serre (Serre [12]).

THEOREM (HOPF). If $G$ is a compact connected Lie group of rank $k$, then the rational cohomology of $G$ is an exterior algebra on $k$ generators of odd degree.

COROLlARY (SERRE). If $G$ is a compact connected Lie group of rank $k$, then $k=-\chi \pi(G)$, where $\chi \pi$ denotes the rational homotopy Euler characteristic, defined explicitly in $\$ 1.1$ below.

A prominent attempt to generalize the definition of rank has been that of Milnor and Smale, which is the following.

Definition. If $M$ is a differentiable manifold, then rank $(M)=$ the maximal number of linearly independent commuting vector fields on $M=$ the maximal number $k$ such that the additive group $R^{k}$ acts differentiably on $M$, with each orbit of dimension $k$.

This definition has proved to be difficult to work with, mainly because relatively little is known about the actions of noncompact groups. Most of the results have concerned only three-dimensional manifolds. For example, there is the work of Lima [9], in which he proves that the rank of $S^{3}$ is equal to one.

This paper is concerned with the rank of a space, as defined by Wu-Yi Hsiang. This definition, given in $\$ 1.1$ below, is based on compact group actions, namely torus actions, and the actions are not required to be differentiable. The rank is thus defined for all topological spaces. We prove that the Hsiang rank of a compact

Received by the editors April 2, 1971.

AMS 1969 subject classifications. Primary 2240; Secondary 5550, 5540.

Key words and phrases. Rank, torus group, Borel setting, rational homotopy, Postnikov resolution. 
Lie group is equal to its rank as a Lie group. Some results are obtained also for certain homogeneous spaces. These are particularly interesting for, if $G$ is a compact Lie group, and if $H$ is a closed subgroup of $G$, then

$$
-\chi \pi(G / H)=-\chi \pi(G)+\chi \pi(H)=\operatorname{rank}(G)-\operatorname{rank}(H) .
$$

These results were obtained as part of my doctoral dissertation at the University of California, Berkeley, written under the direction of Professor Wu-Yi Hsiang. I would like to express my sincere gratitude to Dr. Hsiang for his encouragement and his guidance, and for the perspicacity of his conjecture.

1.1. Definitions. Let $G$ be a torus, let $X$ be a topological space, and let $\Phi: G \times X \rightarrow X$ be a continuous action of $G$ on $X$ having finitely many orbit types. Then Hsiang, in [7], makes the following definitions.

Definition. Let $G_{x}$ denote the isotropy subgroup of $G$ at $x$, and let $\left(G_{x}\right)_{0}$ denote the identity component of $G_{x}$. Then set

$$
\begin{aligned}
r k_{0}(\Phi) & =\min \left\{\operatorname{corank}\left(\left(G_{x}\right)_{0}\right): x \in X\right\} \\
& =\min \left\{\operatorname{rank}\left(G /\left(G_{x}\right)_{0}\right): x \in X\right\} .
\end{aligned}
$$

(Since $G$ is a torus, rank and dimension are synonymous.)

Definition. $r k_{0}(X)=\max \left\{r k_{0}(\Phi)\right\}$, where $\Phi$ ranges over all continuous torus actions on $X$, having finitely many orbit types.

$r k_{0}$ may be called the zero-rank, the connected rank, the toric rank, or, when there is no source of confusion, just the rank. In [7], Hsiang defines simultaneously the $p$-rank of an action and of a space; this is done analogously to the above using actions of elementary $p$-groups for prime $p$.

By the rational homotopy groups of $X$, we shall mean, as usual, the groups $\pi_{i}(X) \otimes Q, i \geqq 1$, where $Q$ denotes the rationals, and where $\pi_{1}(X)$ has been made abelian, if necessary. Then the rank of $\pi_{i}(X)$ as an abelian group, rank $\left(\pi_{i}(X)\right)$, is equal to the dimension of $\pi_{i}(X) \otimes Q$ as a rational vector space, $\operatorname{dim}\left(\pi_{i}(X) \otimes Q\right)$.

Definition. If $X$ has finitely generated homotopy groups, which are purely torsional except in finitely many dimensions, then we shall say that $X$ has finitely generated rational homotopy (FGRH), and we shall define the homotopy (Euler) characteristic of $X$ as

$$
\chi \pi(X)=\sum_{i \geq 1}(-1)^{i} \operatorname{dim}\left(\pi_{i}(X) \otimes Q\right) .
$$

(This is $-\chi L_{*}(X)$, where $L_{*}(X)$ is the rational homotopy Lie algebra of $X$, with its usual grading.)

Let $F \rightarrow E \rightarrow B$ be a fibration with base space $B$, total space $E$, and fibre $F$, all three being connected. By considering the rational homotopy exact sequence of the fibration, it is clear that, if any two of $F, E$ and $B$ have FGRH, then so does the third, and $\chi \pi(E)=\chi \pi(F)+\chi \pi(B)$.

In the following we shall use sheaf theoretic cohomology (Bredon [3]). Later we shall restrict our attention to a class of spaces on which sheaf theoretic cohomology and singular cohomology are equivalent. We consider closed supports only. 
If $X$ has cohomology of finite type over the integers, let $P_{0}(X, t)$ denote the rational Poincaré series of $X$ in indeterminate $t$. That is,

$$
P_{0}(X, t)=\sum_{n \geqq 0} \operatorname{dim}\left(H^{n}(X ; Q)\right) t^{n}
$$

By $P_{0}(Y, t) \leqq P_{0}(X, t)$, we mean that $\operatorname{dim}\left(H^{n}(Y ; Q)\right) \leqq \operatorname{dim}\left(H^{n}(X ; Q)\right)$, for each $n$.

Definition. If $X$ has integral cohomology of finite type, then we define $\rho_{0}(X)$ to be the order of the pole of $P_{0}(X, t)$ at $t=1$. Specifically

$$
\rho_{0}(X)=\inf \left\{\alpha:(1-t)^{\alpha} P_{0}(X, t) \rightarrow 0 \text { as } t \rightarrow 1-\right\} .
$$

( $t$ tends to one from below through real values, and $\rho_{0}(X)$ is defined only if the radius of convergence of $P_{0}(X, t)$ is at least one.)

For example, consider $C P^{\infty}$, infinite complex projective space. $P_{0}\left(C P^{\infty}, t\right)$ $=\sum_{n \geqq 0} t^{2 n}=\left(1-t^{2}\right)^{-1}$. Thus $\rho_{0}\left(C P^{\infty}\right)=1$.

If $\rho_{0}(X)$ is defined and if $P_{0}(Y, t) \leqq P_{0}(X, t)$, then $\rho_{0}(Y)$ is defined, and $\rho_{0}(Y)$ $\leqq \rho_{0}(X)$. Furthermore, if $F \rightarrow E \rightarrow B$ is a fibration, oriented in the sense of Spanier [13], and $\rho_{0}(F)$ and $\rho_{0}(B)$ are defined, then so is $\rho_{0}(E)$, and $\rho_{0}(E) \leqq \rho_{0}(B)+\rho_{0}(F)$. To see this, let $E_{r}^{p, q}$ denote the rational cohomology Leray-Serre spectral sequence of the fibration, and let $E_{r}^{n}=\sum_{p+q=n} E_{r}^{p, q}$.

Then

$$
\begin{aligned}
P_{0}(E, t) & =\sum_{n \geq 0} \operatorname{dim}\left(E_{\infty}^{n}\right) t^{n} \\
& \leqq \sum_{n \geqq 0} \operatorname{dim}\left(E_{2}^{n}\right) t^{n}=P_{0}(B, t) P_{0}(F, t) .
\end{aligned}
$$

Hence $\rho_{0}(E)$ exists, and $\rho_{0}(E) \leqq \rho_{0}(B)+\rho_{0}(F)$.

1.2. Propositions. Let $G$ be a compact topological group which acts on $X$. Then, as in [2], we have an $N$-universal bundle for $G, G \rightarrow E_{G}^{N} \rightarrow B_{G}^{N}$; a universal bundle for $G, G \rightarrow E_{G} \rightarrow B_{G}$; and the induced spaces $X_{G}^{N}=\left(X \times E_{G}^{N}\right) / G$ and $X_{G}=\left(X \times E_{G}\right) / G$ together with the associated bundles $X \rightarrow X_{G}^{N} \rightarrow B_{G}^{N}$ and $X \rightarrow X_{G} \rightarrow B_{G}$.

Following Bredon [4], we set

$$
H^{*}\left(B_{G} ; Q\right)=\operatorname{proj} \lim H^{*}\left(B_{G}^{N} ; Q\right)
$$

and

$$
H^{*}\left(X_{G} ; Q\right)=\text { proj } \lim H^{*}\left(X_{G}^{N} ; Q\right) .
$$

Suppose that $G$ is a torus, and let $\Phi: G \times X \rightarrow X$ denote the action of $G$ on $X$. Suppose, furthermore, that $X$ is compact, that $X$ has finite-dimensional rational cohomology, and that $\operatorname{dim}_{Q}(X)$ is finite, in the sense of Cohen [5]. Then in [7] Hsiang proves the following crucial theorem.

THEOREM 1.2.1 (HSIANG). If $\Phi$ has a finite number of orbit types, then $r k_{0}(\Phi)$ $=\rho_{0}\left(B_{G}\right)-\rho_{0}\left(X_{G}\right)$. 
A similar result for $p$-rank is included in [7]. Of course, in our case, $\rho_{0}\left(B_{G}\right)$ $=\operatorname{dim} G$.

With the same restrictions on $X$, in [7], Hsiang also notes the following.

Proposition 1.2.2. If $\chi(X) \neq 0$, then $r k_{0}(X)=0$. The corresponding result holds for p-rank, when $\chi(X) \not \equiv 0$ modulo $p$.

The proof is by Floyd's formula, that, if $\Phi$ is an elementary p-group action on such a space $X$, with finitely many orbit types, and with fixed point set $F$, then $\chi(X) \equiv \chi(F)$ modulo $p$, and $\chi(X)=\chi(F)$ when $p=0$.

1.3. Conjectures. Let $K$ denote the class of compact connected finite-dimensional metrizable locally contractible topological spaces. Then $K$ coincides with the class of compact connected finite-dimensional metrizable absolute neighborhood retracts, and $K$ contains the class of compact connected $\mathrm{CW}$-complexes. Furthermore, any space in $K$ is dominated by a finite $\mathrm{CW}$-complex, and, hence, any simply-connected space in $K$ has the homotopy type of a finite CW-complex (viz. $\mathrm{Hu}[8])$.

The class $K$ has two properties which are very useful to us. First, from Bredon [3], it follows that sheaf theoretic cohomology and singular cohomology coincide on $K$. Secondly, if $X$ is in $K$ and if $G$ is a torus acting on $X$ with finitely many orbit types, then the orbit space $X / G$ is in $K$. The compactness and connectedness of $X / G$ are obvious, and the metrizability is easy and is known under more general conditions (Palais in [2]). The finite dimensionality of $X / G$ is also true more generally (Montgomery and Yang [11], Palais [2]). Finally, the local contractibility of $X / G$ in these circumstances is a result of Conner [6].

The following three conjectures play an important role in the study of rank. The first is owing to Hsiang [7].

Conjecture $\left(^{*}\right)$. If $X$ is in $K$, and if $X$ has FGRH, then $r k_{0}(X) \leqq-\chi \pi(X)$.

Conjecture $\left({ }^{* *}\right)$. If $X$ is a path-connected topological space, with FGRH, then $\rho_{0}(X) \geqq \chi \pi(X)$. (In this case $\rho_{0}(X)$ is defined in terms of singular cohomology.)

Conjecture $\left(^{* * *}\right)$. If $X$ is in $K$, and if $X$ has FGRH, then $\chi \pi(X) \leqq 0$.

It is clear that $\left({ }^{* *}\right)$ implies $\left({ }^{* * *}\right)$, and that $\left(^{*}\right)$ implies $\left({ }^{* * *}\right)$. We shall show that $\left({ }^{*}\right)$ and $\left({ }^{* * *}\right)$ are equivalent for simply-connected spaces.

2. Relations between the conjectures. In this section, we establish the equivalence of the first and third conjectures, and make some definitions suited to the study of the second.

2.1. Canonical actions. By an almost-free action of a compact Lie group on a space is meant an action for which all the isotropy subgroups are finite.

Observation (Hsiang). If $X$ has rank $r$, then we can find an action, $\Phi$, of the $r$-torus on $X$, which is almost free. That is, $\exists \Phi: T^{r} \times X \rightarrow X$ such that $r k_{0}(\Phi)$ $=r k_{0}(X)=r$.

Proof. Let $\Psi: T^{k} \times X \rightarrow X, k \geqq r$, have rank $r$. Since there are only finitely many 
orbit types, there are only finitely many isotropy subgroups $T_{i} \subseteq T^{k}$; and for each $i$, $\operatorname{dim} T_{i} \leqq k-r$. It is now easy to find a connected closed subgroup of $T^{k}$, say $H$, of dimension $r$, such that $H$ intersects the identity component of each $T_{i}$ in the identity alone.

Definition. If $X$ has rank $r$, and if $\Phi$ is an almost-free action of the $r$-torus on $X$, then we shall say that $\Phi$ is a canonical action on $X$.

In general we shall denote the fibre bundle $X \rightarrow X_{G} \rightarrow B_{G}$ by $\xi(\Phi)$, and the fibre bundle $G \rightarrow X \times E_{G} \rightarrow X_{G}$ by $\eta(\Phi)$. If $\Phi$ is canonical we shall write $\xi(X)$ and $\eta(X)$ for $\xi(\Phi)$ and $\eta(\Phi)$, respectively. There will be no loss of generality through failing to indicate the particular canonical action giving rise to $\xi(X)$ or $\eta(X)$.

By Theorem 1.2.1, if $\Phi$ is canonical, then $\rho_{0}\left(X_{G}\right)=0$.

THEOREM 2.1.1. $\left(^{*}\right)$ is true for simply-connected spaces if and only if $(* * *)$ is true for simply-connected spaces.

Proof. Since, by definition, $r k_{0}(X) \geqq 0$, the implication $\left({ }^{*}\right) \Rightarrow\left({ }^{* *}\right)$ is clear.

To show the reverse implication, let $X$ be a simply-connected space in $K$, with FGRH, whose rank is $r$, and consider a canonical $r$-torus action on $X$.

Let $q$ denote the projection $X_{G} \rightarrow X^{\prime}$, where $X^{\prime}=X / G$ is the orbit space. Then, for any $y \in X^{\prime}, q^{-1}(y)=B_{G_{y}}$ is $Q$-acyclic, as $G_{y}$ is finite. By the Vietoris-Begle Mapping Theorem, therefore, $q^{*}: H^{*}\left(X^{\prime} ; Q\right) \rightarrow H^{*}\left(X_{G} ; Q\right)$ is an isomorphism. $X^{\prime}$ is in $K$, and so the cohomology can be singular. Hence $q_{*}: H_{*}\left(X_{G} ; Q\right)$ $\rightarrow H_{*}\left(X^{\prime} ; Q\right)$ is an isomorphism; and by the Whitehead-Serre Theorem, modulo the class of torsional abelian groups, $X_{G}$ and $X^{\prime}$ have isomorphic rational homotopy groups. Thus, from $\xi(X), X^{\prime}$ has FGRH, and by assumption of $\left(^{* * *}\right), \chi \pi\left(X^{\prime}\right)$ $\leqq 0$; and so $\chi \pi\left(X_{G}\right) \leqq 0$, too.

From $\xi(X), \chi \pi\left(X_{G}\right)=\chi \pi(X)+\chi \pi\left(B_{G}\right)=\chi \pi(X)+r$. Hence $r k_{0}(X)=r \leqq-\chi \pi(X)$.

REMARKS. (1) $X^{\prime}$, above, is simply-connected as a result of Montgomery and Yang [11].

(2) The Vietoris-Begle isomorphism was obtained as a limit from the maps $q^{N}: X_{G}^{N^{\prime}} \rightarrow X^{\prime}$, which give rise to isomorphisms, $\left(q^{N}\right)^{*}$, in degrees less than $N$.

(3) If $r k_{0}(X) \leqq-\chi \pi(X)$ for all one-connected finite CW-complexes with FGRH, then $r k_{0}(X) \leqq-\chi \pi(X)$ for all simply-connected spaces in $K$ with FGRH; that is, $\left(^{*}\right)$ is true for simply-connected spaces. For our premise implies that $\chi \pi(X) \leqq 0$ for all one-connected finite $\mathrm{CW}$-complexes with FGRH, and hence $\chi \pi(X) \leqq 0$ for all spaces having the homotopy type of a one-connected finite $\mathrm{CW}$-complex with FGRH. In particular, then, $\left({ }^{* * *}\right)$ is true for simply-connected spaces.

For convenience we introduce the following definitions.

Definitions. (i) A space $X$ has form I if $\pi_{i}(X) \otimes Q \neq 0 \Rightarrow i$ is odd.

(ii) A space $X$ has form II if $\pi_{i}(X) \otimes Q \neq 0 \Rightarrow i$ is even.

(iii) A space $X$ has form III if $\pi_{i}(X) \otimes Q \neq 0$ and $\pi_{j}(X) \otimes Q \neq 0$ and $i$ is even and $j$ is odd $\Rightarrow i<j$. 
(iv) A space $X$ has form IV if $\pi_{i}(X) \otimes Q \neq 0$ and $\pi_{j}(X) \otimes Q \neq 0$ and $i$ is even and $j$ is odd $\Rightarrow j<i$.

(v) A space $X$ has form V if $\pi_{i}(X) \otimes Q \neq 0$ and $\pi_{j}(X) \otimes Q \neq 0$ and $\pi_{k}(X) \otimes Q \neq 0$ and $i$ is even and $k$ is even and $i \leqq j \leqq k \Rightarrow j$ is even.

Clearly a space having form I, II, III or IV also has form V.

The following proposition enables us to deduce results about the rank of a space having a certain form from results about the homotopy characteristic of a space having a related form.

Proposition 2.1.2. Given a torus action, $\Phi: G \times X \rightarrow X$, on a one-connected space $X$, then we have the following.

(a) $X$ has form I $\Rightarrow X_{G}$ has form III.

(b) $X$ has form II $\Rightarrow X_{G}$ has form II.

(c) $X$ has form III $\Rightarrow X_{G}$ has form III.

Proof. Consider the homotopy exact sequence of $\xi(\Phi)$. If $G=T^{m}$, then $B_{G}$ $=K\left(Z^{m}, 2\right)$, and so $\pi_{i}\left(X_{G}\right) \cong \pi_{i}(X)$ for $i \geqq 3$. Also $X_{G}$ is one-connected, and finally we have the short exact sequence

$$
0 \rightarrow \pi_{2}(X) \rightarrow \pi_{2}\left(X_{G}\right) \rightarrow Z^{m} \rightarrow 0 .
$$

This completes the proof.

COROLlaRY 2.1.3. If $\left({ }^{* *}\right)$ is true for one-connected spaces with FGRH of form II or III, then $\left(^{*}\right)$ is true for simply-connected spaces of form I, II or III.

Proof. Let $X$ be in $K$, simply-connected with FGRH of form I, II or III. Consider a canonical $r$-torus action on $X$, where $r k_{0}(X)=r$.

From $\xi(X), X_{G}$ is one-connected with FGRH. By Proposition 2.1.2, $X_{G}$ has form II or III, and by Theorem 1.2.1, $\rho_{0}\left(X_{G}\right)=0 . \rho_{0}$ is with respect to singular cohomology since $X$, and hence all $X_{G}^{N}$, are in $K$.

By our assumption of $\left({ }^{* *}\right)$, therefore, $\chi \pi\left(X_{G}\right) \leqq 0$.

The result now follows from $\xi(X)$.

2.2. An upper bound for $r k_{0}(X)$. In this section we obtain an upper bound for the rank of a space, $X$, in $K$. In the case when $X$ has finitely generated rational homotopy, this upper bound is, in general, far less stringent than the upper bound conjectured in $(*)$.

By $m(X ; Q)=k$, for $k$ a nonnegative integer, we mean that $H^{k}(X ; Q) \neq 0$, but that $H^{n}(X ; Q)=0$ whenever $n$ is greater than $k$.

Proposition 2.2.1. Let $X$ be a space in $K$. Suppose that $r k_{0}(X)=r$, and that $X_{G}$ is obtained from a canonical r-torus action on $X\left(G=T^{r}\right)$. Suppose further that $m(X ; Q)=n$, and that $m\left(X_{G} ; Q\right)=k$. Then $k+r=n$.

Proof. Since $\eta(X)$ is induced from the fibration $G \rightarrow E_{G} \rightarrow B_{G}$, it is orientable 
in the sense of Spanier [13]. Hence we may apply the Serre spectral sequence in rational cohomology to $\eta(X)$,

$$
E_{2}^{k, r} \cong H^{k}\left(X_{G} ; Q\right) \otimes H^{r}(G ; Q) \neq 0,
$$

and clearly $E_{2}^{k, r}=E_{\infty}^{k, r}$. The result is now immediate, since $m(G ; Q)=r$.

Corollary 2.2.2. $r k_{0}(X) \leqq m(X ; Q)$.

Proposition 2.2.3. Suppose that $X$ is in $K$, that $m(X ; Q)=n$, and that $X$ is $2 k$-connected or $(2 k-1)$-connected. Then $r k_{0}(X) \leqq n-2 k$.

Proof. In the spectral sequence of $\xi(X)$ we see that either connectivity condition implies that $E_{\infty}^{2 k, 0}=E_{2}^{2 k, 0} \neq 0$.

Hence $m\left(X_{G} ; Q\right) \geqq 2 k$, and the result follows from Proposition 2.2.1.

REMARK. In the statement of the above proposition we could replace the conditions " $X$ is $2 k$-connected or $(2 k-1)$-connected" by " $\tilde{H}^{i}(X ; Q)=0$ for $0 \leqq i \leqq 2 k$ or $2 k-1$ " respectively.

By $X \sim Y$ over $Q$ we mean that $H^{*}(X ; Q)$ and $H^{*}(Y ; Q)$ are isomorphic as graded rational vector spaces.

COROLLARY 2.2.4. Let $N=\left\{n_{i}: 1 \leqq i \leqq k\right\}$ be a finite nondecreasing sequence of positive integers. Let $N$ (even) be the number of even integers in $N$, and let $N$ (odd) be the number of odd integers in $N$. Suppose that $X \sim \bigvee_{i=1}^{k} S^{n_{i}}$ over $Q$, where $\bigvee_{i=1}^{k} S^{n_{1}}$ is the pointed sum of the spheres, $S^{n_{i}}$, and suppose that $X$ is in $K$.

Then

(i) if $n_{1}$ is even, $r k_{0}(X) \leqq n_{k}-n_{1}$,

(ii) if $n_{1}$ is odd, $r k_{0}(X) \leqq n_{k}-n_{1}+1$, and

(iii) if $N$ (odd) $\neq N$ (even) $+1, r k_{0}(X)=0$.

Proof. (i) and (ii) are immediate from Proposition 2.2.3. (iii) follows from Proposition 1.2.2.

It is worth noting that, if $k \geqq 2$, the space $X$ of Corollary 2.2.4 does not have finitely generated rational homotopy.

The procedure of comparing the spectral sequence of $\xi(X)$ with that of $\eta(X)$ may be continued to provide more detailed strictures on the size of the rank than those of Proposition 2.2.3. For example, it is easy to show that, if $X \sim S^{2} \vee S^{3} \vee S^{5}$ over $Q$, and if $X$ is in $K$, then $r k_{0}(X) \leqq 1$. The referee has pointed out that there exists such an $X$ with rank one. Let $X$ be the result of joining an $S^{3}$ to an $S^{5}$ along an $S^{1}$. Then $X$ has the homotopy type of $S^{2} \vee S^{3} \vee S^{5}$, and $r k_{0}(X)=1$.

3. On the Conjecture (**). The purpose of this section is to prove the truth of $\left({ }^{* *}\right)$ for one-connected spaces having form V. The proof will follow from a Postnikov resolution of the space in question.

3.1. Orders of pole in principal fibrations. Let $\pi$ be a finitely generated abelian group of rank $k$, and let $K(\pi, n), n>1$, be the Eilenberg-Mac Lane space. Let 
$\xi=(E, B, p)$ be a principal fibration with base space $B$, total space $E$, induced from the path-loop fibration over $K(\pi, n)$. Then we have the following proposition.

Proposition 3.1.1. (i) If $n$ is even, then $\rho_{0}(B) \geqq \rho_{0}(E) \geqq \rho_{0}(B)-k$.

(ii) If $n$ is odd, then $\rho_{0}(B)+k \geqq \rho_{0}(E) \geqq \rho_{0}(B)$.

Proof. We have the following fibre square:

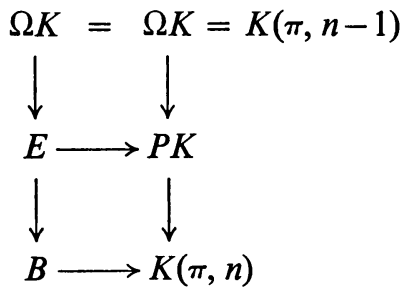

Up to homotopy type, $E \rightarrow B \rightarrow K(\pi, n)$ is a fibration.

From the Serre spectral sequence in rational cohomology of the fibration $\Omega K \rightarrow E \rightarrow B$, it follows that $\rho_{0}(E) \leqq \rho_{0}(B)+\rho_{0}(\Omega K)$. If $n$ is even, then $\rho_{0}(\Omega K)=0$, and if $n$ is odd, then $\rho_{0}(\Omega K)=k$.

From the Serre spectral sequence in rational cohomology of the fibration $E \rightarrow B \rightarrow K(\pi, n)$, it follows that $\rho_{0}(B) \leqq \rho_{0}(E)+\rho_{0}(K(\pi, n))$. If $n$ is even, then $\rho_{0}(K(\pi, n))=k$, and if $n$ is odd, then $\rho_{0}(K(\pi, n))=0$.

This completes the proof.

REMARK. If $n$ is odd, if $B$ is one-connected, and if $K(\pi, n) \rightarrow E \rightarrow B$ is a fibration, which is not necessarily principal, then we can still deduce that $\rho_{0}(B) \geqq \rho_{0}(E)$ $\geqq \rho_{0}(B)-k$. The first inequality is clear, and the second inequality may be inferred by applying a spherical class resolution (Mahowald [10] or Thomas [14]) and using the Serre spectral sequence at each stage.

3.2. $\left(^{* *}\right)$ For spaces of form V. First we prove $\left({ }^{* *}\right)$ for spaces of form IV.

LEMMA 3.2.1. Let $X$ be a one-connected space with finitely generated rational homotopy and having form IV. Then $\rho_{0}(X) \geqq \chi \pi(X)$.

Proof. Let $\pi_{2 n+1}(X)$ be the highest homotopy group of $X$ of odd dimension and nonzero rank; and let $\pi_{2 m}(X)$ be the highest homotopy group of $X$ of nonzero rank $(m \geqq n+1)$.

Let

$$
b=\sum_{i=1}^{2 n+1} \operatorname{rank}\left(\pi_{i}(X)\right) \text { and } a=\sum_{i=2 n+2}^{2 m} \operatorname{rank}\left(\pi_{i}(X)\right)
$$

so that $\chi \pi(X)=a-b$.

Let $F \rightarrow X_{2 n+1} \rightarrow X$ be a $(2 n+1)$-connective fibration over $X$. Then, by the exact sequence of rational homotopy, both $F$ and $X_{2 n+1}$ have form II, and $\chi \pi(F)$ $=b$, and $\chi \pi\left(X_{2 n+1}\right)=a$. 
Now, if $Y$ is a space having finitely generated rational homotopy and having form II, and if $\chi \pi(Y)=c$, then let us consider the Postnikov resolution of $Y$. If we look at the Serre spectral sequence in rational cohomology at each stage of the resolution, then it becomes clear that $H^{*}(Y ; Q)$ is a polynomial algebra on $c$ generators of even degree. Hence $\rho_{0}(Y)=c$.

Thus $\rho_{0}\left(X_{2 n+1}\right)=a$ and $\rho_{0}(F)=b$. But from the Serre spectral sequence of $F \rightarrow X_{2 n+1} \rightarrow X$, it is clear that $\rho_{0}\left(X_{2 n+1}\right) \leqq \rho_{0}(X)+\rho_{0}(F)$. Therefore, $\rho_{0}(X) \geqq a-b$, which completes the proof.

REMARK. If $Y$ has finitely generated rational homotopy, if $Y$ is one-connected, and if $Y$ has form $\mathrm{I}$, then, by considering the Postnikov resolution of $Y$, it follows that $\rho_{0}(Y)=0 \geqq \chi \pi(Y)$.

Proposition 3.2.2. Let $X$ be a one-connected space having finitely generated rational homotopy and having form $\mathrm{V}$. Then $\rho_{0}(X) \geqq \chi \pi(X)$.

Proof. Suppose that the nonzero rational homotopy groups of $X, \pi_{i}(X) \otimes Q$, are odd dimensional for $i \leqq 2 m+1$, even dimensional for $2 m+2 \leqq i \leqq 2 n$, and odd dimensional for $i \geqq 2 n+1$ (form $\mathrm{V}$ ).

Let

$$
a=\sum_{i \leqq 2 m+1} \operatorname{rank}\left(\pi_{i}(X)\right), \quad b=\sum_{2 m+2 \leqq i \leqq 2 n} \operatorname{rank}\left(\pi_{i}(X)\right),
$$

and

$$
c=\sum_{i \geqq 2 n+1} \operatorname{rank}\left(\pi_{i}(X)\right)
$$

Thus $\chi \pi(X)=-a+b-c$.

Consider the Postnikov resolution of $X$, and let $E_{2 n}$ be the space at the stage where the first $2 n$ homotopy groups of $X$ have been taken into account. There is a fibration $F \rightarrow X \rightarrow E_{2 n} . E_{2 n}$ has form IV, and $\chi \pi\left(E_{2 n}\right)=-a+b . F$ has form I, and $\chi \pi(F)=-c$.

Each stage of the resolution of $F \rightarrow X \rightarrow E_{2 n}$ is a principal fibration of the form $K\left(\pi_{r-1}(F), r-1\right) \rightarrow E \rightarrow B$, induced from the path loop fibration over $K\left(\pi_{r-1}(F), r\right)$. Since $F$ has form I, and since $X$ has FGRH, $\pi_{r-1}(F) \otimes Q$ is zero, except for finitely many even values of $r$, and $\sum_{r} \operatorname{dim}\left(\pi_{r}(F) \otimes Q\right)=c$. By Proposition 3.1.1(i),

$$
\rho_{0}(E) \geqq \rho_{0}(B)-\operatorname{dim}\left(\pi_{r-1}(F) \otimes Q\right),
$$

and so, after all relevant stages have been taken into account, we have that $\rho_{0}(X)$ $\geqq \rho_{0}\left(E_{2 n}\right)-c$.

But, by Lemma 3.2.1, $\rho_{0}\left(E_{2 n}\right) \geqq-a+b$.

REMARK. The situation $\rho_{0}(X)>\chi \pi(X)$ is possible. For example, if $X=K(Z, 3)$ $\times K(Z, 4)$, then $\rho_{0}(X)=1$, but $\chi \pi(X)=0$.

COROLlaRY 3.2.3. If $X$ is in $K$, simply-connected with FGRH of form I or III, then $r k_{0}(X) \leqq-\chi \pi(X)$. 
Proof. Immediate from Corollary 2.1.3.

We conclude this section by considering a space $X$, whose rational homotopy groups fall into a stable range. That is, if $\pi_{n}(X) \otimes Q$ is the nonzero rational homotopy group of lowest dimension, and if $\pi_{m}(X) \otimes Q$ is the one of highest dimension, then $m \leqq 2 n-2$. The following proposition is a trivial consequence of the Postnikov resolution.

Proposition 3.2.4. If $X$ is a one-connected space with FGRH, whose rational homotopy groups fall into a stable range, then

$$
\rho_{0}(X)=\sum_{i \text { even }} \operatorname{dim}\left(\pi_{i}(X) \otimes Q\right) .
$$

4. Applications. In this section we use the above results to study the rank of certain interesting spaces.

4.1. Lie groups and $H$-spaces.

THEOREM 4.1.1. Let $X$ be an $H$-space in $K$. Then $r k_{0}(X) \leqq-\chi \pi(X)$.

Proof. Since $X$ is dominated by a finite CW-complex, it has finitely generated rational cohomology groups. By the Leray structure theorem for Hopf algebras, then, $H^{*}(X ; Q)$ is an exterior algebra on finitely many generators of odd degree.

Now, by Serre [12], there is a one-to-one correspondence between the generators of $H^{*}(X ; Q)$ and the generators of the rational homotopy groups of $X$, which preserves degree.

If $X$ were simply-connected, then the result would follow immediately from Corollary 3.2.3. In general, however, $X$ is homotopy simple; that is, $\pi_{1}(X)$ acts trivially on all homotopy groups of $X$. Thus, if $\Phi$ is a given torus action on $X$, there exists a Moore-Postnikov resolution of $\xi(\Phi)$. The result now follows from Proposition 3.1.1 and Theorem 1.2.1.

THEOREM 4.1.2. Let $G$ be a compact connected Lie group of rank $k$. Then $r k_{0}(G)=k$.

Proof. By Serre [12], $k=-\chi \pi(G)$. By Theorem 4.1.1, therefore, $r k_{0}(G) \leqq k$.

But a maximal torus of $G, T^{k}$ acts freely on $G$ by left or right translations, and so $r k_{0}(G) \geqq k$.

4.2. Some homogeneous spaces. Homogeneous spaces make excellent testing spaces for the conjecture (*). For, if $G$ is a compact Lie group of rank $k$, and if $U$ is a closed subgroup of rank $r$, then a maximal torus of $G$ acts on $G / U$ with rank $k-r$. Thus $r k_{0}(G / U) \geqq k-r$. At the same time, from the homotopy exact sequence of the fibre bundle $U \rightarrow G \rightarrow G / U$, we have that $-\chi \pi(G / U)=-\chi \pi(G)+\chi \pi(U)$, and hence, $-\chi \pi(G / U)=k-r$.

We exhibit below some elementary homogeneous spaces to which Corollary 3.2.3 is applicable. 
First, however, we note that if $k=r$, then $r k_{0}(G / U)=0$. Borel shows in [1] that, under such circumstances, $H^{i}(G / U ; Q)$ is zero for all odd $i$. The result then follows from Proposition 1.2.2. Thus, for example, the toric rank is zero for real, complex or quaternionic Grassmann manifolds.

Proposition 4.2.1. If $G$ is a compact one-connected Lie group of rank $k$, and if $T$ is a toric subgroup of $G$ of rank $r$, then $r k_{0}(G / T)=k-r$.

Proof. Clearly $G / T$ has form III.

Proposition 4.2.2. For $1 \leqq k \leqq n-m, r k_{0}(U(n) /(U(m) \times U(k)))=n-m-k$.

Proof. $U(n) /(U(m) \times U(k))$ is one-connected with form III.

Likewise, where $S G_{n, k}(C)=U(n) /(U(n-k) \times S U(k))$ is the variety of oriented $k$-planes in complex $n$-space, $r k_{0}\left(S G_{n, k}(C)\right)=1$. Similar considerations apply to the real and quaternionic cases.

The following also is inferred by an easy Moore-Postnikov resolution argument, using Theorem 1.2.1 directly.

Proposition 4.2.3. If $G$ is a compact one-connected Lie group of rank $k$, and if $U$ is a closed connected subgroup of rank 1 (i.e. $U$ is isomorphic to $S^{1}$, SO (3) or $\operatorname{Sp}(1))$, then $r k_{0}(G / U)=k-1$.

The remainder of this section will be devoted to Stiefel manifolds. $V_{n, k}(F)$, for $F=R, C$ or $H$, will denote the Stiefel manifold of $k$-frames in real, complex or quaternionic $n$-space, respectively.

THEOREM 4.2.4. (a) If $n \geqq 3$, and $0 \leqq k \leqq n-3$, then $r k_{0}\left(V_{n, k}(R)\right)=[k / 2]+1$, if $n$ is even and $k$ is odd, and $r k_{0}\left(V_{n, k}(R)\right)=[k / 2]$, otherwise. $([x]=$ greatest integer less than or equal to $x$.)

(b) If $0 \leqq k \leqq n$, then $r k_{0}\left(V_{n, k}(C)\right)=k$.

(c) If $0 \leqq k \leqq n$, then $r k_{0}\left(V_{n, k}(H)\right)=k$.

Proof. (a) Since $k<n, V_{n, k}(R)=\mathrm{SO}(n) / \mathrm{SO}(n-k)$, and, since $n \geqq 3$ and $n-k \geqq 3$, $V_{n, k}(R)$ is one-connected.

Now, by the homotopy exact sequence, we have that $V_{n, k}(R)$ has form I if $n-k$ is odd, and $V_{n, k}(R)$ has form III if $n-k$ is even.

Hence, in either case,

$$
r k_{0}\left(V_{n, k}(R)\right) \leqq-\chi \pi\left(V_{n, k}(R)\right)=[n / 2]-[(n-k) / 2] .
$$

(b) $V_{n, k}(C)=U(n) / U(n-k)$. This has form I, and the result follows.

(c) $V_{n, k}(H)=\mathrm{Sp}(n) / \mathrm{Sp}(n-k)$, and again the results follow at once.

4.3. Products of spheres. 
THEOREM 4.3.1. Let $m_{1}, \ldots, m_{k}$ be a nondecreasing sequence of positive even integers with $m_{k} \leqq 2 m_{1}-2$. Let $n_{1}, \ldots, n_{r}$ be positive odd integers, and let $n=\min \left\{n_{1}, \ldots, n_{r}\right\}$. Suppose that $n>m_{k}$ and let $X=S^{m_{1}} \times \cdots \times S^{m_{k}} \times S^{n_{1}} \times \cdots$ $\times S^{n_{r}}$. Then $r k_{0}(X)=r$.

Proof. Under the given conditions $X$ is a one-connected CW-complex with FGRH of form III; or, in the event that no even integers are given, $X$ is a homotopy simple CW-complex with FGRH of form I. In either case, $\chi \pi(X)=-r$.

When there are even integers, then, by Corollary 3.2.3, $r k_{0}(X) \leqq r$.

When there are no even integers (and $n$ may be one), $r k_{0}(X) \leqq r$ by a MoorePostnikov resolution of $\xi(\Phi)$ for any torus action $\Phi$ on $X$ and Theorem 1.2.1.

Now if $S^{2 n-1}$ is an odd-dimensional sphere, viewing it as the unit sphere in complex $n$-space, the circle group acts freely on it in the obvious way. Hence $r k_{0}(X) \geqq r$.

REMARK. If no odd integers are given, then the condition that $m_{k} \leqq 2 m_{1}-2$ may be dropped; for, $r k_{0}\left(S^{m_{1}} \times \cdots \times S^{m_{k}}\right)=0$ by Proposition 1.2.2.

The rank of complex or quaternionic projective space is zero by Proposition 1.2.2. For the same reason, the rank of even dimensional real projective space is zero. We conclude with the following.

THEOREM 4.3.2. Let $R P^{n}$ denote real projective $n$-space, and let $n_{1}, \ldots, n_{r}$ be positive odd integers. Let $X=R P^{n_{1}} \times \cdots \times R P^{n_{r}}$. Then $r k_{0}(X)=r$.

Proof. $X$ is homotopy simple, $X$ has FGRH of form I, and $\chi \pi(X)=-r$. As before, therefore, $r k_{0}(X) \leqq r$.

But the free action of $S^{1}$ on $S^{2 n-1}$ induces an almost-free action (every isotropy subgroup is $Z_{2}$ ) on $R P^{2 n-1}$. Hence $r k_{0}(X) \geqq r$.

\section{REFERENCES}

1. A. Borel, Sur la cohomologie des espaces fibrés principaux et des espaces homogènes de groupes de Lie compacts, Ann. of Math. (2) 57 (1953), 115-207. MR 14, 490.

2 A. Borel et al., Seminar on transformation groups, Ann. of Math. Studies, no. 46, Princeton Univ. Press, Princeton, N. J., 1960. MR 22 \#7129.

3. G. E. Bredon, Sheaf theory, McGraw-Hill, New York, 1967. MR 36 \#4552.

4. - Cohomological aspects of transformation groups, Proc. Conf. on Transformation Groups (New Orleans, La., 1967), Springer, New York, 1968, pp. 245-280. MR 39 \#6303.

5. H. Cohen, $A$ cohomological definition of dimension for locally compact Hausdorff spaces, Duke Math. J. 21 (1954), 209-224. MR 16, 609.

6. P. E. Conner, Retraction properties of the orbit space of a compact topological transformation group, Duke Math. J. 27 (1960), 341-357. MR 29 \#1286.

7. W.-Y. Hsiang, On generalizations of a theorem of A. Borel and their applications in the study of topological actions, University of California, Berkeley, 1969 (preprint).

8. S.-T. Hu, Theory of retracts, Wayne State University Press, Detroit, 1965. MR 31 \#6202.

9. E. L. Lima, Commuting vector fields on $S^{3}$, Ann. of Math. (2) 81 (1965), 70-81. MR 30 \#1517. 
10. M. E. Mahowald, On obstruction theory in orientable fiber bundles, Trans. Amer. Math. Soc. 110 (1964), 315-349. MR 28 \#620.

11. D. Montgomery and C.-T. Yang, The existence of a slice, Ann. of Math. (2) 65 (1957), 108-116. MR 19, 291.

12. J.-P. Serre, Groupes d'homotopie et classes de groupes abéliens, Ann. of Math. (2) 58 (1953), 258-294. MR 15, 548.

13. E. H. Spanier, Algebraic topology, McGraw-Hill, New York, 1966. MR 35 \#1007.

14. E. Thomas, Seminar on fiber spaces, Lecture Notes in Math., no. 13, Springer-Verlag, Berlin, 1966. MR 34 \#3582.

Department of Mathematics, University of Hawail, Honolulu, Hawail 96822 